\title{
EDITORIAL AND COMMENT Language Barriers, Informed Consent, and Effective Caregiving
}

\author{
Lachlan Forrow, MD and Jane Crandall Kontrimas, BS CoreCHITM
}

Division of General Medicine and Primary Care, Beth Israel Deaconess Medical Center, Boston, MA, USA.

J Gen Intern Med 32(8):855-7

DOI: $10.1007 / \mathrm{s} 11606-017-4068-0$

(c) Society of General Internal Medicine 2017

The treatment of a disease may be entirely impersonal; the care of a patient must be completely personal. Francis W. Peabody (1927) ${ }^{1}$

In this issue of JGIM, Lee et al. report on an important study about a crucial aspect of the care of patients with limited English proficiency (LEP), demonstrating both sobering shortcomings in current practice and the potential for modest but important improvements through simple steps that are within the capability of every health facility. ${ }^{2}$ While their study addresses a single, narrowly focused aspect of caring for LEP patients, it should cause all of us to reflect more broadly on the importance of understanding the barriers to care these populations face, and of energetically pursuing quality improvement strategies.

The narrow problem that the investigators address is the challenge of obtaining informed consent for invasive inpatient medical procedures for LEP patients. The limited in-person availability of professional interpreters in most hospitals, and the proven limitations of using others (often bilingual family members) to interpret, makes this a daunting challenge. ${ }^{3}$ At the same time, proceeding with an invasive medical procedure in the absence of informed consent violates one of the most fundamental norms - both legal and ethical — of medical practice, and is thus clearly unacceptable except possibly in a lifethreatening emergency. ${ }^{4}$

The intervention that Lee et al. designed and evaluated was both simple and easily generalizable - installing and promoting the use of dual-handset interpreter telephones at every inpatient bedside, with $24 / 7$ availability of professional telephone interpretation. In their pre/post evaluation, they used a brief patient-centered assessment of the adequacy of informed consent, asking each patient three questions: (1) How well do you understand the reasons you need to have the procedure/ surgery? (2) How well do you understand the risks of the procedure/surgery? (3) Did you get all of your questions answered? Possible answers to the first two questions were "very well", "well", "not well", or "not at all", while the third

Published online May 10, 2017 question required a "yes" or "no" response from patients. Consent was considered "adequately informed" if patients answered "very well" to the first two questions and "yes" to the third. Rates of consent considered "adequate" increased from just $29 \%$ in the pre-intervention period to $54 \%$ postintervention, compared to $74 \%$ for English-speaking patients.

There are obvious limitations to the use of this measure for assessing whether consent was "adequately informed" - patient-perceived adequacy of medical information provided does not demonstrate that the information provided was complete, accurate, or unbiased, or that the patient actually understood essential elements. On the other hand, more robust assessments, or even a different scoring system, might determine that consent was "adequately informed" more frequently than the authors report, since in their assessment, if a patient reported that he or she understood the risks only "well" (rather than "very well"), consent was considered not "adequately informed." In addition, achieving "informed consent" is far more complex than effectively transmitting information, and is misconceived as a discrete "event" rather than an ongoing process of dialogue, reflection, and decisionmaking within a clinician-patient relationship. ${ }^{5}$ Much more thorough assessments of the content, process, and impact of informed consent could have been very helpful, not only in assessing the impact of the intervention, but in understanding more fully the variety of barriers to achieving informed consent with LEP patients, so that we can more reliably and successfully address them. ${ }^{6}$

Several features of the study raise the possibility of both unidentified bias favoring positive results and uncertain generalizability to other settings: its pre/post-evaluation design, the lack of a concurrent control, the limited duration of the study (8 months), and its conduct within a single institution. But these limitations should not distract from the authors' convincing evidence that a simple intervention with nearuniversal generalizability achieved a major improvement in patient experience: dual handset telephones can be installed at any point of care, anywhere in our health system, and can be used for any interactions with patients with limited English proficiency. While the primary language of all patients enrolled in the study was either Spanish or Chinese, the authors point out that "the phone had programmed buttons enabling 24-hour access to remote professional interpreters for more than 100 languages in less than one minute." Their study thus 
has potential implications for practice far beyond the narrow issue of informed consent for inpatient invasive procedures for Chinese- and Spanish-speaking patients.

The authors observe that "over 25 million people in the United States...have limited English proficiency (LEP)", and that previous studies have demonstrated that these patients experience "higher error and readmission rates in the hospital setting and less health education and healthcare utilization in the ambulatory setting." There can be little doubt that these well-documented problems of patient safety and quality of care are also associated with substantial unnecessary costs of care. For example, while the current paper does not provide data on the financial implications of the intervention, a separate, recently published paper from the same study reports a significant reduction in 30-day readmission rates for the LEP group during the 8-month intervention period compared with the 18 months pre-intervention $(13.4 \%$ post-intervention vs. $17.8 \%$ pre-intervention), at the same time that Englishproficient patient readmission rates at the same hospital increased slightly. ${ }^{7}$ The authors of that paper estimated monthly hospital expenditure savings, after subtracting the cost of the telephone interpreter services $(\$ 0.99 / \mathrm{min} \times 28,284$ total minutes, or $\$ 28,001$ total cost) of $\$ 161,404$ ( $\$ 1,291,233$ total for the 8-month period). If even a small fraction of these savings can be confirmed in future studies, the financial benefits alone of the intervention would make it compelling. It is unlikely that the benefits found in this study are as easy to achieve as simply installing dual-handset interpreter telephones at every bedside. As the authors stress, the intervention also included a vigorous hospital-wide promotional effort that included meetings with all hospital nurse managers to plan the implementation and communication with nursing staff at all staff meetings, daily rounds, and through floor-specific newsletters, as well as outreach regarding the phones to all clinical chiefs of service, who in turn communicated by email with their attending and resident physicians. The authors report that prior to the intervention, each floor already had 1-3 dual-handset interpreter-phones available on mobile carts or in locked cabinets, suggesting a major benefit from the improved convenience of having phones already at each bedside. It is plausible, however, that the promotional efforts were as crucial to the intervention's success as the easier availability of the telephones. To the extent that these promotional efforts boosted efforts by nurses and physicians to improve communication with LEP patients generally, the measured improvements in patientreported understanding may not be fully attributable to the specific "informed consent" conversations utilizing the dualhandset telephones. It is unlikely that the separately reported finding of reduced overall readmission rates for all LEP patients during the intervention period was entirely, or even primarily, attributable to improvements in a single informed consent interaction for a single invasive procedure for the subset of patients undergoing one.
It would be tragically misguided to assume from this study that universal bedside (or office) availability of dual-handset interpreter telephones is the key to improving the care of LEP patients more generally. What is needed to achieve informed consent for an invasive procedure for any patient has very limited relevance to what is needed for effective ongoing care of that patient. Even in the acute care setting, the contributions of interpreters to patient care extend far beyond assistance with verbal communication, including helping with cross-cultural understanding that may be crucial to the development and implementation of an effective care plan, and that is far more likely to develop through in-person collaboration than through telephone support from an essentially anonymous voice.

In the outpatient setting, most medical issues involve chronic conditions whose successful management depends on establishing an effective longitudinal partnership with the patient. As Francis Peabody observed nearly a century ago, "The essence of the practice of medicine is that it is an intensely personal matter...The significance of the intimate personal relationship between physician and patient cannot be too strongly emphasized, for in an extraordinarily large number of cases both diagnosis and treatment are directly dependent on it." While in some settings, professional interpreters may be expected to limit their role to the interpretation of only the words spoken (which is largely achievable over a telephone), it is our experience as a physician and professional interpreter collaborating for over 30 years that a broader view of clinician-interpreter partnerships can be invaluable in fostering the longitudinal personal relationships between patients and doctors that effective care often depends on.

The care of patients across language barriers can be very difficult, and is often a source of major frustration both for patients and for the clinicians who try to care for them. But when effective clinician-interpreter partnerships succeed, the care of LEP patients can be wonderfully rewarding, not just when it leads to improved patient outcomes, but through the deeply personal human relationships with patients that, for both clinicians and interpreters, is often the most cherished part of our work. ${ }^{8}$

Corresponding Author: Lachlan Forrow, MD; Division of General Medicine and Primary Care, Beth Israel Deaconess Medical Center, 330 Brookline Avenue, Bosotn, MA 02215, USA (e-mail: Lforrow@bidmc.harvard.edu).

\section{Compliance with Ethical Standards:}

Conflict of Interest: The authors declare that they do not have conflict of interest.

Disclosure: Dr. Forrow reports no conflict of interest. Ms. Kontrimas reports no financial conflict of interest, but notes that she is a member of the National Council on Interpreting in Health Care: "The NCIHC is a multidisciplinary organization whose mission is to promote and enhance language access in health care in the United States." 


\section{REFERENCES}

1. Peabody FW. The Care of the Patient. JAMA. 2015;313(18):1868.

2. JS Lee, EJ Pérez-Stable, SE Gregorich, et al. Increased Access to Professional Interpreters in the Hospital Improves Informed Consent for Patients with Limited English Proficiency. J Gen Intern Med. (2017) DOI: 10.1007/s11606-017-3983-4

3. Flores G, Abreu M, Barone CP, Bachur R, Lin $\mathbf{H}$. Errors of medical interpretation and their potential clinical consequences: a comparison of professional versus ad hoc versus no interpreters. Ann Emerg Med. 2012;60(5):545-553.

4. Berg JW, Appelbaum PS, Lidz CW, Parker L. Informed consent: legal theory and clinical practice. 2nd ed. New York: Oxford University Press, 2001.
5. Lidz CW, Appelbaum PS, Meisel A. Two models of implementing informed consent. Arch Intern Med. 1988;148(6):1385-9.

6. Kinnersley $\mathbf{P}$, Phillips $\mathbf{K}$, Savage $\mathbf{K}$ et al. Interventions to promote informed consent for patients undergoing surgical and other invasive healthcare procedures. Cochrane Database Syst Rev. 2013;(7).

7. Karliner LS, Pérez-Stable EJ, Gregorich SE. Convenient Access to Professional Interpreters in the Hospital Decreases Readmission Rates and Estimated Hospital Expenditures for Patients With Limited English Proficiency. Med Care. 2017;55(3):199-206.

8. Srivastava R. The Interpreter. N Engl J Med 2017; 376:812-813 